\title{
CONFORMALITY OF QUASICONFORMAL MAPPINGS AT A POINT, REVISITED
}

\author{
Mitsuhiro Shishikura \\ Kyoto University, Department of Mathematics \\ Kyoto 606-8502, Japan; mitsu@math.kyoto-u.ac.jp
}

\begin{abstract}
We present a new and simple proof of Teichmüller-Wittich-Belinskiı̌'s and Gutlyanski1-Martio's theorems on the conformality of quasiconformal mappings at a given point. Known proofs gave separate estimates for the radial and angular variations, but our proof unifies them using Grötzsch-type inequality for the variation of cross-ratio of four points on the Riemann sphere. We also give a sufficient condition for $C^{1+\alpha}$-conformality.
\end{abstract}

\section{Introduction}

Quasiconformal mappings are known to be differentiable almost everywhere with respect to the Lebesgue measure (see [A1], [LV]). However if one picks a specific point, then the differentiability is not guaranteed. In this paper, we discuss the conformality (i.e. the differentiability with zero $\bar{z}$-derivative) of quasiconformal mappings at a given point.

Definition. For a quasiconformal mapping $f: \mathbf{C} \rightarrow \mathbf{C}$, we denote

$$
\mu_{f}(z)=\frac{f_{\bar{z}}(z)}{f_{z}(z)}=\frac{\frac{\partial f}{\partial \bar{z}}}{\frac{\partial f}{\partial z}}, \quad K_{f}(z)=\frac{1+\left|\mu_{f}(z)\right|}{1-\left|\mu_{f}(z)\right|} \quad \text { and } \quad K(f)=\operatorname{ess} \sup K_{f}(z) .
$$

We say that $f$ is conformal at $z=z_{0}$ if the limit

$$
f^{\prime}\left(z_{0}\right)=\lim _{z \rightarrow z_{0}} \frac{f(z)-f\left(z_{0}\right)}{z-z_{0}}
$$

exists and is non-zero. For simplicity, we only discuss the conformality at $z=0$, but the conformality at other points can be treated similarly by translating the coordinate.

There is a well-known criterion for the pointwise conformality:

Theorem 1. (Teichmüller [T], Wittich [W], Belinskiı̌ [B1], Lehto [L1]; see [LV, Theorem 6.1]) If $f$ is a quasiconformal mapping satisfying

$$
\frac{1}{2 \pi} \iint_{|z|<r} \frac{\left|\mu_{f}(z)\right|}{|z|^{2}} d x d y<\infty \text { for some } r<\infty,
$$

then $f$ is conformal at $z=0$.

This theorem was improved by:

https://doi.org/10.5186/aasfm.2018.4359

2010 Mathematics Subject Classification: Primary 30C62.

Key words: Quasiconformal mapping.

This work was partially supported by JSPS Grant-in-Aid 26287016 and 15K13444. 
Theorem 2. (Gutlyanskiǔ-Martio $[\mathrm{GM}]$ ) Let $f: \mathbf{C} \rightarrow \mathbf{C}$ be a quasiconformal mapping. If

$$
\iint_{|z|<1} \frac{\left|\mu_{f}(z)\right|^{2}}{1-\left|\mu_{f}(z)\right|^{2}} \frac{d x d y}{|z|^{2}}<\infty
$$

and the limit

$$
\lim _{r \searrow 0} \iint_{r<|z|<1} \frac{\mu_{f}(z)}{1-\left|\mu_{f}(z)\right|^{2}} \frac{d x d y}{z^{2}}
$$

exists, then $f$ is conformal at $z=0$.

The goal of this paper is to give a new and simple proof of this theorem. (Note that in $[\mathrm{GM}]$, it was assumed that (2) holds without $1-\left|\mu_{f}(z)\right|^{2}$ in the denominator, but this is equivalent for a qc-mapping.) The proof of Theorem 1 consists of the differentiability of the absolute value $|f(z)|$ (e.g. Teichmüller [T], Wittich [W]; see [LV] Lemma 6.1), and the estimate the variation of $\arg \frac{f(z)}{z}$ (e.g. Belinskiı̌ [B1], Lehto [L1]; see [LV] Lemma 6.2). The proof of Theorem 2 in [GM] also gave the estimates for the absolute value and the argument.

Our approach unifies the two estimates into the form of the variation of cross-ratio of four points $0, z_{1}, z_{2}, \infty$, via Cauchy's criterion (see Lemmas 4 and 5 ). The effect of quasiconformal mapping is usually measured by the integral of $\mu_{f}$ paired with a suitable quadratic differential. In our case, the quadratic differential to consider is $\varphi_{z_{1}, z_{2}}(z) d z^{2}$, where

$$
\varphi_{z_{1}, z_{2}}(z)=\frac{z_{1}}{z\left(z-z_{1}\right)\left(z-z_{2}\right)}
$$

The quasiconformal variation of cross-ratio is formulated in Theorem 6 , and the Main Theorem 8 is stated in terms of the integral $J\left(\mu ; z_{1}, z_{2}\right)$ defined by (11) using $\varphi_{z_{1}, z_{2}}$. Heuristically when $\left|z_{2}\right| \ll\left|z_{1}\right|$, in the annular region in-between, $\left|z_{2}\right| \ll|z| \ll$ $\left|z_{1}\right|$, the quadratic differential $\varphi_{z_{1}, z_{2}}(z) d z^{2}$ "looks like" $c \frac{d z^{2}}{z^{2}}$, and this explains the appearance of $\frac{1}{z^{2}}$ in Theorems 1 and 2. (See [HSS], for a decomposition theorem of quadratic differentials, in which this idea was extensively used.) This observation will be justified by the estimates on integrals (Lemmas 10 and 11) via the decomposition (21).

Moreover we can also derive a more quantitative estimate on the remainder term:

Theorem 3. Let $f: \mathbf{C} \rightarrow \mathbf{C}$ be a quasiconformal mapping and suppose that

$$
I(r)=\iint_{\{z:|z|<r\}} \frac{\left|\mu_{f}(z)\right|}{1-\left|\mu_{f}(z)\right|^{2}} \frac{d x d y}{|z|^{2}}
$$

is finite and has order $O\left(r^{\beta}\right)(r \searrow 0)$ for some $\beta>0$. Then for any $0<\alpha<\frac{\beta}{2+\beta}, f$ is $C^{1+\alpha}$-conformal at 0 in the sense that

$$
f(z)=f(0)+f^{\prime}(0) z+O\left(|z|^{1+\alpha}\right) \quad \text { as } z \rightarrow 0 .
$$

Remark. Schatz $[\mathrm{S}]$ obtained a similar result by assuming a stronger conditipn $\iint \frac{|\mu(z)|}{|z|^{p}} d x d y<\infty(p>2)$, which implies (5) with $\beta=p-2$. McMullen [McM] (Theorem 2.25) obtained the same conclusion by assuming $\operatorname{Area}\left(B_{r}(0) \cap \operatorname{supp} \mu\right)=$ $O\left(r^{2+\alpha}\right)$, which is again stronger.

For further references, see also [RW], [D], [BJ].

The author would like to thank Kari Astala, David Drasin, Frederick Gardiner, Anatoly Golberg for helpful discussions. 


\section{Conformality at $z=0$ and cross-ratio}

We start with nothing but Cauchy's criterion. Define the cylinder $\mathcal{C}=\mathbf{C} / 2 \pi i \mathbf{Z}$ and its distance $|w|_{\mathcal{C}}=\inf \{|w+2 \pi i n|: n \in \mathbf{Z}\}$.

Lemma 4. Let $f: \mathbf{C} \rightarrow \mathbf{C}$ be an orientation-preserving homeomorphism with $f(0)=0$ and fix a constant $0<\delta_{1} \leq 1$. Then the following are equivalent:

(a) $f$ is conformal at $z=0$;

(b) there exists a limit $\lim _{z \rightarrow 0} \log \frac{f(z)}{z}$ for a suitable choice of branch of $\log$;

(c) for any $\varepsilon>0$, there exists $r>0$ such that if $0<\left|z_{1}\right|<r$ and $0<\left|z_{2}\right| \leq \delta_{1}\left|z_{1}\right|$, then

$$
\left|\log \frac{f\left(z_{1}\right)}{z_{1}}-\log \frac{f\left(z_{2}\right)}{z_{2}}\right|_{\mathcal{C}}<\varepsilon
$$

Proof. The most of implications are obvious, and we only prove that (c) implies (b). First take $\varepsilon=\frac{\pi}{2}$, then (c) implies that the variation of the argument of $\frac{f(z)}{z}$ is less than $\pi$ when $z$ is small. In such a case, the distance $|\cdot|_{\mathcal{C}}$ in $(7)$ can be replaced by the Euclidean distance. Now take smaller $\varepsilon$, and let $r$ be as in (c). If $\left|z_{1}\right|,\left|z_{2}\right|<r$, then take the third point $z_{3}$ so that $\left|z_{3}\right| \leq \delta_{1}\left|z_{1}\right|,\left|z_{3}\right| \leq \delta_{1}\left|z_{2}\right|$, then

$$
\left|\log \frac{f\left(z_{1}\right)}{z_{1}}-\log \frac{f\left(z_{2}\right)}{z_{2}}\right|_{\mathcal{C}} \leq\left|\log \frac{f\left(z_{1}\right)}{z_{1}}-\log \frac{f\left(z_{3}\right)}{z_{3}}\right|_{\mathcal{C}}+\left|\log \frac{f\left(z_{2}\right)}{z_{2}}-\log \frac{f\left(z_{3}\right)}{z_{3}}\right|_{\mathcal{C}}<2 \varepsilon .
$$

By Cauchy's criterion, we have (b). (In fact, for any sequence $z_{n} \rightarrow 0,\left\{\log \frac{f\left(z_{n}\right)}{z_{n}}\right\}$ will be a Cauchy sequence in $\mathcal{C}$, hence it is convergent, and this implies the convergence of $\log \frac{f(z)}{z}$ as $z \rightarrow 0$.)

Definition. For distinct points $z_{1}, z_{2}, z_{3}, z_{4}$ in $\mathbf{C}$, define the cross-ratio by

$$
\operatorname{Cr}\left(z_{1}, z_{2}, z_{3}, z_{4}\right)=\frac{z_{1}-z_{3}}{z_{2}-z_{3}} \cdot \frac{z_{2}-z_{4}}{z_{1}-z_{4}}
$$

This definition extends to the case where one of $z_{j}$ 's is $\infty$ by taking the limit. The cross-ratio belongs to the three punctured sphere $\Omega:=\widehat{\mathbf{C}} \backslash\{0,1, \infty\}=\mathbf{C} \backslash\{0,1\}$. Denote the hyperbolic distance on $\Omega$ by $d_{\Omega}(\cdot, \cdot)$, which is induced from $\frac{|d z|}{\operatorname{Im} z}$ on the universal cover $\mathbf{H}$.

Let $f$ be as in Lemma 4 and take $z_{1}, z_{2} \in \mathbf{C} \backslash\{0\}$ with $z_{1} \neq z_{2}$. Denote $\zeta_{1}=\frac{z_{2}}{z_{1}}=\operatorname{Cr}\left(z_{1}, z_{2}, \infty, 0\right)$ and $\zeta_{2}=\frac{f\left(z_{2}\right)}{f\left(z_{1}\right)}=\operatorname{Cr}\left(f\left(z_{1}\right), f\left(z_{2}\right), \infty, 0\right)$. We need to estimate

$$
\begin{aligned}
\left|\log \frac{f\left(z_{1}\right)}{z_{1}}-\log \frac{f\left(z_{2}\right)}{z_{2}}\right|_{\mathcal{C}} & =\left|\log \zeta_{1}-\log \zeta_{2}\right|_{\mathcal{C}} \\
& =\left|\log \operatorname{Cr}\left(z_{1}, z_{2}, \infty, 0\right)-\log \operatorname{Cr}\left(f\left(z_{1}\right), f\left(z_{2}\right), \infty, 0\right)\right|_{\mathcal{C}}
\end{aligned}
$$

Lemma 5. For any $L>0$, there exist constants $C_{1}>0$ and $0<\delta_{1}<1$ such that if $\zeta_{1}, \zeta_{2} \in \Omega=\mathbf{C} \backslash\{0,1\}$ satisfy $\left|\zeta_{1}\right|<\delta_{1}$ and $d_{\Omega}\left(\zeta_{1}, \zeta_{2}\right) \leq L$, then

$$
\left|\log \zeta_{1}-\log \zeta_{2}\right|_{\mathcal{C}} \leq C_{1} d_{\Omega}\left(\zeta_{1}, \zeta_{2}\right) \cdot \log \frac{1}{\left|\zeta_{1}\right|}
$$

Proof. Let $\rho_{\Omega}(\zeta)|d \zeta|$ be the hyperbolic metric of $\Omega$. It is well-known (see [A2] $\S 1-8)$ that there exist $0<\delta_{0}<1$ and $C_{0}>0$ such that

$$
\rho_{\Omega}(\zeta) \geq \frac{C_{0}}{|\zeta| \log \frac{1}{|\zeta|}} \quad \text { for } 0<|\zeta| \leq \delta_{0}
$$


Let $\nu=e^{L / C_{0}}(>1)$. Then for $0<r \leq \delta_{0}$, the distance between $\{\zeta:|\zeta|=r\}$ and $\left\{\zeta^{\prime}:\left|\zeta^{\prime}\right|=r^{\nu}\right\}$ is bounded below by

$$
d_{\Omega}\left(\zeta, \zeta^{\prime}\right) \geq C_{0} \int_{r^{\nu}}^{r} \frac{d s}{s \log \frac{1}{s}}=C_{0}\left(-\log \log \frac{1}{r}+\log \log \frac{1}{r^{\nu}}\right)=C_{0} \log \nu=L .
$$

Let $\delta_{1}=\delta_{0}^{\nu}$ and $C_{1}=\frac{\nu}{C_{0}}$. Suppose $0<\left|\zeta_{1}\right| \leq \delta_{1}$ and $d_{\Omega}\left(\zeta_{1}, \zeta_{2}\right) \leq L$, and let $\gamma$ be the shortest hyperbolic geodesic in $\Omega$ joining $\zeta_{1}$ and $\zeta_{2}$. Then, by the above estimate for the circles of radii $\left|\zeta_{1}\right|^{\nu},\left|\zeta_{1}\right|,\left|\zeta_{1}\right|^{1 / \nu}$, we have for $\zeta \in \gamma,\left|\zeta_{1}\right|^{\nu} \leq|\zeta| \leq\left|\zeta_{1}\right|^{1 / \nu} \leq \delta_{0}$. Hence

$$
\begin{aligned}
\left|\log \zeta_{1}-\log \zeta_{2}\right|_{\mathcal{C}} & \leq\left|\int_{\gamma} \frac{d \zeta}{\zeta}\right| \leq \int_{\gamma} \frac{|d \zeta|}{|\zeta|} \leq \frac{1}{C_{0}} \int_{\gamma} \rho_{\Omega}(\zeta) \log \frac{1}{|\zeta|}|d \zeta| \\
& \leq \frac{\nu}{C_{0}} \log \frac{1}{\left|\zeta_{1}\right|} \int_{\gamma} \rho_{\Omega}(\zeta)|d \zeta|=C_{1} d_{\Omega}\left(\zeta_{1}, \zeta_{2}\right) \cdot \log \frac{1}{\left|\zeta_{1}\right|}
\end{aligned}
$$

Thus, in order to to show the conformality, we want to show that $d_{\Omega}\left(\zeta_{1}, \zeta_{2}\right) \cdot \log \frac{1}{\left|\zeta_{1}\right|}$ is small when $z_{1}, z_{2}$ are small.

\section{Grötzsch-type inequality for cross-ratio variation}

We need the following Grötzsch-type inequality for cross-ratio variation.

Theorem 6. Let $f: \widehat{\mathbf{C}} \rightarrow \widehat{\mathbf{C}}$ be a quasiconformal mapping and $z_{1}, z_{2}, z_{3}, z_{4}$ distinct points in $\widehat{\mathbf{C}}$, and put $z_{j}^{\prime}=f\left(z_{j}\right)(j=1,2,3,4)$. Then

$$
d_{\Omega}\left(\operatorname{Cr}\left(z_{1}, z_{2}, z_{3}, z_{4}\right), \operatorname{Cr}\left(z_{1}^{\prime}, z_{2}^{\prime}, z_{3}^{\prime}, z_{4}^{\prime}\right)\right) \leq \log \bar{K}_{f}\left(z_{1}, z_{2}, z_{3}, z_{4}\right),
$$

where

$$
\bar{K}_{f}\left(z_{1}, z_{2}, z_{3}, z_{4}\right):=\frac{\sup _{\theta \in \mathbf{R}} \iint_{\mathbf{C}} \frac{\left|1+e^{i \theta} \mu(z) \frac{\varphi(z)}{|\varphi(z)|}\right|^{2}}{1-|\mu(z)|^{2}}|\varphi(z)| d x d y}{\iint_{\mathbf{C}}|\varphi(z)| d x d y}
$$

with $\mu(z)=\mu_{f}(z)$ and $\varphi(z)=\frac{1}{\left(z-z_{1}\right)\left(z-z_{2}\right)\left(z-z_{3}\right)\left(z-z_{4}\right)}$ (omit $\left(z-z_{j}\right)$ if $z_{j}=\infty$ ).

This is a special case of Fundamental Inequality in the Teichmüller theory [GL, Chap. 4, Theorem 9] applied to four punctured sphere. In fact, this case can be proven directly as in [A1]. For the completeness, we will outline this proof in Appendix A.

Note that the above inequality implies the classical Grötzsch inequality

$$
d_{\Omega}\left(\operatorname{Cr}\left(z_{1}, z_{2}, z_{3}, z_{4}\right), \operatorname{Cr}\left(z_{1}^{\prime}, z_{2}^{\prime}, z_{3}^{\prime}, z_{4}^{\prime}\right)\right) \leq \log K(f),
$$

since $\bar{K}_{f}\left(z_{1}, z_{2}, z_{3}, z_{4}\right) \leq K(f)$. We now express $\bar{K}_{f}$ in terms of the following integrals.

Definition. Let $z_{1}, z_{2} \in \mathbf{C} \backslash\{0\}$ with $z_{1} \neq z_{2}$. Note that $\left|\varphi_{z_{1}, z_{2}}\right|$ is integrable over C. Let

$$
J_{*}\left(z_{1}, z_{2}\right)=\iint_{\mathbf{C}}\left|\varphi_{z_{1}, z_{2}}(z)\right| d x d y .
$$

For a measurable function $\mu: \mathbf{C} \rightarrow \mathbf{C}$ with $\|\mu(z)\|_{\infty}<1$, define

$$
J\left(\mu ; z_{1}, z_{2}\right)=2\left|\iint_{\mathbf{C}} \frac{\mu(z) \varphi_{z_{1}, z_{2}}(z)}{1-|\mu(z)|^{2}} d x d y\right|+2 \iint_{\mathbf{C}} \frac{|\mu(z)|^{2}\left|\varphi_{z_{1}, z_{2}}(z)\right|}{1-|\mu(z)|^{2}} d x d y .
$$


Lemma 7. Suppose $0<\left|z_{2}\right|<\left|z_{1}\right|$. Then for $f$ in Theorem 6 , we have

$$
\begin{aligned}
J_{*}\left(z_{1}, z_{2}\right) & \geq 2 \pi \frac{1}{\left|1-\frac{z_{2}}{z_{1}}\right|} \log \frac{\left|z_{1}\right|}{\left|z_{2}\right|} ; \\
\bar{K}_{f}\left(z_{1}, z_{2}, 0, \infty\right) & =1+\frac{J\left(\mu_{f} ; z_{1}, z_{2}\right)}{J_{*}\left(z_{1}, z_{2}\right)} ; \\
J\left(\mu_{f} ; z_{1}, z_{2}\right) & \leq \iint_{\mathbf{C}}\left(K_{f}(z)-1\right)\left|\varphi_{z_{1}, z_{2}}(z)\right| d x d y \leq(K(f)-1) J_{*}\left(z_{1} \cdot z_{2}\right) .
\end{aligned}
$$

Proof. Denote $\varphi=\varphi_{z_{1}, z_{2}}$. By the Residue Theorem, we have for $\left|z_{2}\right|<r<\left|z_{1}\right|$,

$$
\begin{aligned}
\int_{|z|=r} z \varphi(z) d z & =\int_{|z|=r} \frac{z_{1}}{\left(z-z_{1}\right)\left(z-z_{2}\right)} d z \\
& =2 \pi i \operatorname{Res}_{z=z_{2}} \frac{z_{1}}{\left(z-z_{1}\right)\left(z-z_{2}\right)}=2 \pi i \frac{z_{1}}{z_{2}-z_{1}} .
\end{aligned}
$$

Hence $2 \pi\left|\frac{z_{1}}{z_{1}-z_{2}}\right|=\left|\int_{0}^{2 \pi} r e^{i \theta} \varphi\left(r e^{i \theta}\right) i r e^{i \theta} d \theta\right| \leq r^{2} \int_{0}^{2 \pi}\left|\varphi\left(r e^{i \theta}\right)\right| d \theta$ and

$$
\begin{aligned}
J_{*}\left(z_{1}, z_{2}\right) & \geq \int_{\left\{\left|z_{2}\right|<|z|<\left|z_{1}\right|\right\}}|\varphi(x+i y)| d x d y=\int_{\left|z_{2}\right|}^{\left|z_{1}\right|} \int_{0}^{2 \pi}\left|\varphi\left(r e^{i \theta}\right)\right| r d \theta d r \\
& \geq 2 \pi\left|\frac{z_{1}}{z_{1}-z_{2}}\right| \int_{\left|z_{2}\right|}^{\left|z_{1}\right|} \frac{d r}{r}=2 \pi \frac{1}{\left|1-\frac{z_{2}}{z_{1}}\right|} \log \frac{\left|z_{1}\right|}{\left|z_{2}\right|}
\end{aligned}
$$

The equality for $\bar{K}_{f}$ is obvious from

$$
\left|1+e^{i \theta} \mu_{f}(z) \frac{\varphi(z)}{|\varphi(z)|}\right|^{2}=\left(1-\left|\mu_{f}(z)\right|^{2}\right)+2 \operatorname{Re}\left(e^{i \theta} \mu_{f}(z) \frac{\varphi(z)}{|\varphi(z)|}\right)+2\left|\mu_{f}(z)\right|^{2} .
$$

The last inequality follows from $2 \frac{\left|\mu_{f}(z)\right|+\left|\mu_{f}(z)\right|^{2}}{1-\left|\mu_{f}(z)\right|^{2}}=\frac{1+\left|\mu_{f}(z)\right|}{1-\left|\mu_{f}(z)\right|}-1 \leq K_{f}(z)-1$.

\section{Main Theorem and Proof of Theorems 2 and 3}

Our criterion for the pointwise conformality is as follows:

Theorem 8. Let $f: \mathbf{C} \rightarrow \mathbf{C}$ be a $K$-quasiconformal mapping with $f(0)=0$ and suppose that there exists $0<\delta<1$ such that

$$
J\left(\mu_{f} ; z_{1}, z_{2}\right) \rightarrow 0 \text { when } z_{1} \text { and } z_{2} \text { tend to } 0 \text { satisfying } 0<\left|z_{2}\right| \leq \delta\left|z_{1}\right| .
$$

Then $f$ is conformal at $z=0$. Moreover there exists a constant $C>0$ depending only on $K$ such that

$$
\left|\log \frac{f(z)}{z}-\log f^{\prime}(0)\right|_{\mathcal{C}} \leq C \liminf _{z_{2} \rightarrow 0} J\left(\mu_{f} ; z, z_{2}\right) .
$$

This follows from the following lemma.

Lemma 9. (Key Inequality) Given $K>1$, there exist $0<\delta_{1}<1$ and $C>0$ such that if $f: \mathbf{C} \rightarrow \mathbf{C}$ is a $K$-quasiconformal mapping with $f(0)=0$, then for $0<\left|z_{2}\right| \leq \delta_{1}\left|z_{1}\right|$

$$
\left|\log \frac{f\left(z_{1}\right)}{z_{1}}-\log \frac{f\left(z_{2}\right)}{z_{2}}\right|_{\mathcal{C}} \leq C J\left(\mu_{f} ; z_{1}, z_{2}\right)
$$


Proof. Let $\delta_{1}<1$ and $C_{1}$ be as in Lemma 5 for $L=\log K$. Then take $C=$ $\frac{C_{1}\left(1+\delta_{1}\right)}{2 \pi}$. For two distinct points $z_{1}, z_{2} \in \mathbf{C} \backslash\{0\}$ and consider the cross-ratios $\zeta_{1}=\frac{z_{2}}{z_{1}}=\operatorname{Cr}\left(z_{1}, z_{2}, \infty, 0\right)$ and $\zeta_{2}=\frac{f\left(z_{2}\right)}{f\left(z_{1}\right)}=\operatorname{Cr}\left(f\left(z_{1}\right), f\left(z_{2}\right), \infty, 0\right)$. By Theorem 6 and Lemma 7 and $\log (1+x) \leq x(x \geq 0)$, we have

$$
d_{\Omega}\left(\zeta_{1}, \zeta_{2}\right) \leq \log \bar{K}_{f}\left(z_{1}, z_{2}, \infty, 0\right) \leq \frac{J\left(\mu_{f} ; z_{1}, z_{2}\right)}{J_{*}\left(z_{1}, z_{2}\right)} \leq \frac{J\left(\mu_{f} ; z_{1}, z_{2}\right)}{2 \pi \frac{1}{\left|1-\frac{z_{2}}{z_{1}}\right|} \log \frac{\left|z_{1}\right|}{\left|z_{2}\right|}}
$$

By the classical Grötzsch inequality, we have $d_{\Omega}\left(\zeta_{1}, \zeta_{2}\right) \leq \log K=L$. Hence by Lemma 5, if $0<\left|z_{2}\right| \leq \delta_{1}\left|z_{1}\right|$, then we have

$$
\begin{aligned}
\left|\log \frac{f\left(z_{1}\right)}{z_{1}}-\log \frac{f\left(z_{2}\right)}{z_{2}}\right|_{\mathcal{C}} & =\left|\log \frac{\zeta_{1}}{\zeta_{2}}\right|_{\mathcal{C}} \leq C_{1} d_{\Omega}\left(\zeta_{1}, \zeta_{2}\right) \log \frac{1}{\left|\zeta_{1}\right|} \\
& \leq \frac{C_{1}\left|1-\frac{z_{2}}{z_{1}}\right|}{2 \pi} J\left(\mu_{f} ; z_{1}, z_{2}\right) \leq \frac{C_{1}\left(1+\delta_{1}\right)}{2 \pi} J\left(\mu_{f} ; z_{1}, z_{2}\right) \\
& =C J\left(\mu_{f} ; z_{1}, z_{2}\right) .
\end{aligned}
$$

Proof of Theorem 8. This is an immediate consequence of Lemma 9. By the assumption (12), in which we may replace $\delta$ by a smaller one so that $\delta \leq \delta_{1}$, (c) of Lemma 4 holds, hence $f$ is conformal at $z=0$. Moreover taking the limit $z_{2} \rightarrow 0$ in (16), we obtain (13).

In order to deduce Theorem 2 from Theorem 8 , we need to relate $J\left(\mu_{f} ; z_{1}, z_{2}\right)$ to (2) and (3). For this purpose, we define the following quantity.

Definition. Let $p>2$ and $p>s>0$. For $\mu \in L^{\infty}(\mathbf{C})$ with $\|\mu\|_{\infty}<1$, define

$$
I_{p, s}(\mu ; r)=\iint_{\mathbf{C}} \frac{|\mu(z)|^{p}}{\left(1-|\mu(z)|^{2}\right)^{p}} \frac{d x d y}{|z|^{2}\left(1+\frac{|z|}{r}\right)^{s}}
$$

For $0<r<R$, denote $A(r, R)=\{z \in \mathbf{C}: r<|z|<R\}$.

The following two lemmas will be proved in $\S 4$.

Lemma 10. Let $\mu \in L^{\infty}(\mathbf{C})$ with $\|\mu\|_{\infty}<1$. Then for any $p>s>0$ with $p>2$ and $0<\rho<1$, there exists $C^{\prime}=C^{\prime}(p, s, \rho)>0$ such that if $0<\left|z_{2}\right|<\rho^{2}\left|z_{1}\right|$, then

$$
\begin{aligned}
& \left|\iint_{\mathbf{C}} \frac{\mu(z) \varphi_{z_{1}, z_{2}}(z)}{1-|\mu(z)|^{2}} d x d y\right| \\
& \leq \frac{1}{1-\rho^{2}}\left|\iint_{A\left(\rho^{-1}\left|z_{2}\right|, \rho\left|z_{1}\right|\right)} \frac{\mu(z)}{1-|\mu(z)|^{2}} \frac{d x d y}{z^{2}}\right|+C^{\prime} I_{p, s}\left(\mu ;\left|z_{1}\right|\right)^{\frac{1}{p}} \\
& \iint_{\mathbf{C}} \frac{|\mu(z)|^{2}\left|\varphi_{z_{1}, z_{2}}(z)\right|}{1-|\mu(z)|^{2}} d x d y \\
& \leq \frac{1}{1-\rho^{2}} \iint_{A\left(\rho^{-1}\left|z_{2}\right|, \rho\left|z_{1}\right|\right)} \frac{|\mu(z)|^{2}}{1-|\mu(z)|^{2}} \frac{d x d y}{|z|^{2}}+C^{\prime} I_{p, s}\left(\mu ;\left|z_{1}\right|\right)^{\frac{1}{p}}
\end{aligned}
$$

Lemma 11. For $\mu \in L^{\infty}(\mathbf{C})$ with $\|\mu\|_{\infty}<1$ satisfying (2) and for $p>2$ and $p>s>0$, the integral $I_{p, s}(\mu ; r)$ is finite. Moreover there exist constants $C_{2}$ and $C_{3}$ depending only on $K=\frac{1+\|\mu\|_{\infty}}{1-\|\mu\|_{\infty}}$ such that for $0<r<r^{\prime}$,

$$
I_{p, s}(\mu ; r) \leq C_{2} \iint_{\left\{|z|<r^{\prime}\right\}} \frac{|\mu(z)|^{2}}{1-|\mu(z)|^{2}} \frac{d x d y}{|z|^{2}}+\frac{C_{3}}{s}\left(\frac{r}{r^{\prime}}\right)^{s} .
$$


Therefore $I_{p, s}(\mu ; r) \rightarrow 0$ as $r \searrow 0$.

Assuming these lemmas, we can give:

Proof of Theorem 2. Since the convergence in (3) and (2) imply that the first terms on the right hand sides of (18) and (19) tend to 0 as $z_{1} \rightarrow 0$, Theorem 2 follows from Theorem 8 and Lemmas 10, 11.

Proof of Theorem 3. Suppose $I(r)=O\left(r^{\beta}\right)(r \searrow 0)$ and $0<\alpha<\frac{\beta}{2+\beta}$. According to Theorem 8 and Lemma 10, in order to prove (6), it suffices to show that all the terms in (18) and (19) have order $O\left(r^{\alpha}\right)$. This is obvious for the first terms. Choose $s=2$ and $p>2$ so that $\alpha<\frac{2 \beta}{2+\beta} \frac{1}{p}$. Let $\gamma=\frac{2}{2+\beta}$ and take $r^{\prime}=r^{\gamma}$ in Lemma 11. Both terms on the right hand side of $(20)$ have order $O\left(r^{\frac{2 \beta}{2+\beta}}\right)$, hence $I_{p, s}(\mu ; r)^{\frac{1}{p}}=O\left(r^{\alpha}\right)$. Thus $(6)$ is proved.

\section{Estimates on the integrals $J\left(\mu ; z_{1}, z_{2}\right)$ and $I_{p, s}(r)$}

Proof of Lemma 10. Let $\mu, p, s, \rho$ be as in Lemma 10 and suppose $0<\left|z_{2}\right| \leq$ $\rho^{2}\left|z_{1}\right|$. Since

$$
\varphi_{z_{1}, z_{2}}(z)+\frac{z_{1}}{z_{1}+z_{2}} \cdot \frac{1}{z^{2}}=\frac{z_{1}}{z_{1}+z_{2}}\left(\psi_{1}(z)+\psi_{2}(z)\right)
$$

where $\psi_{1}(z)=\frac{1}{\left(z-z_{1}\right)\left(z-z_{2}\right)}$ and $\psi_{2}(z)=\frac{z_{1} z_{2}}{z^{2}\left(z-z_{1}\right)\left(z-z_{2}\right)}$, the decomposition of the integral into $\mathbf{D}\left(\rho^{-1}\left|z_{2}\right|\right)=\left\{z \in \mathbf{C}:|z|<\rho^{-1}\left|z_{2}\right|\right\}, \mathbf{D}^{*}\left(\rho\left|z_{1}\right|\right)=\left\{z \in \mathbf{C}:|z|>\rho\left|z_{1}\right|\right\}$ and $A\left(\rho^{-1}\left|z_{2}\right|, \rho\left|z_{1}\right|\right)$ gives

$$
\begin{aligned}
& \left|\iint_{\mathbf{C}} \frac{\mu(z) \varphi_{z_{1}, z_{2}}(z)}{1-|\mu(z)|^{2}} d x d y+\frac{z_{1}}{z_{1}+z_{2}} \iint_{A\left(\rho^{-1}\left|z_{2}\right|, \rho\left|z_{1}\right|\right)} \frac{\mu(z)}{1-|\mu(z)|^{2}} \frac{d x d y}{z^{2}}\right| \\
& \leq\left|\iint_{\mathbf{D}\left(\rho^{-1}\left|z_{2}\right|\right)} \frac{\mu(z) \varphi_{z_{1}, z_{2}}(z)}{1-|\mu(z)|^{2}} d x d y\right|+\left|\iint_{\mathbf{D}^{*}\left(\rho\left|z_{1}\right|\right)} \frac{\mu(z) \varphi_{z_{1}, z_{2}(z)}(z)}{1-|\mu(z)|^{2}} d x d y\right| \\
& \quad+\frac{1}{\left|1+\frac{z_{2} \mid}{z_{1}}\right|}\left(\left|\iint_{A\left(\rho^{-1}\left|z_{2}\right|, \rho\left|z_{1}\right|\right)} \frac{\mu(z) \psi_{1}(z)}{1-|\mu(z)|^{2}} d x d y\right|\right. \\
& \left.\quad+\left|\iint_{A\left(\rho^{-1}\left|z_{2}\right|, \rho\left|z_{1}\right|\right)} \frac{\mu(z) \psi_{2}(z)}{1-|\mu(z)|^{2}} d x d y\right|\right) .
\end{aligned}
$$

It is easy to see that (18) holds if one can prove that each term on the right hand side of $(22)$ is bounded by $I_{p, s}\left(\mu ;\left|z_{1}\right|\right)^{\frac{1}{p}}$ up to a constant factor. Take $q$ such that $\frac{1}{p}+\frac{1}{q}=1$, then $1<q<2$. For any measurable set $D \subset \mathbf{C}$, denote by $I_{p, s}(\mu ; r, D)$ the integral in (17) with the domain $\mathbf{C}$ replaced by $D$. For a measurable set $D \subset \mathbf{C}$ and an integrable function $\psi(z)$ on $D$, the Hölder inequality yields

$$
\begin{aligned}
& \left|\iint_{D} \frac{\mu(z) \psi(z)}{1-|\mu(z)|^{2}} d x d y\right| \\
& \leq\left.\iint_{D}\left|\frac{\mu(z)}{\left(1-|\mu(z)|^{2}\right)|z|^{\frac{2}{p}}\left(1+\frac{|z|}{r}\right)^{\frac{s}{p}}}\right| \cdot|| z\right|^{\frac{2}{p}}\left(1+\frac{|z|}{r}\right)^{\frac{s}{p}} \psi(z) \mid d x d y \\
& \leq I_{p, s}(\mu ; r, D)^{\frac{1}{p}} H(\psi, r, D)^{\frac{1}{q}},
\end{aligned}
$$


where

$$
H(\psi, r, D)=\iint_{D}|z|^{2 q-2}\left(1+\frac{|z|}{r}\right)^{s(q-1)}|\psi(z)|^{q} d x d y
$$

In order to estimate the terms in (22), we apply (23) with $r=\left|z_{1}\right|, \psi=\varphi_{z_{1}, z_{2}}, \psi_{1}$, $\psi_{2}$, and $\left.D=\mathbf{D}\left(\rho^{-1}\left|z_{2}\right|\right), \mathbf{D}^{*}\left(\rho\left|z_{1}\right|\right)\right), A\left(\rho^{-1}\left|z_{2}\right|, \rho\left|z_{1}\right|\right)$. It suffices to show that the corresponding $H(\psi, r, D)$ is finite.

For the first term of the right hand side of (22), we now give an estimate on $H(\psi, r, D)$ for $\psi(z)=\varphi_{z_{1}, z_{2}}(z)=-\frac{1}{z\left(z-z_{2}\right)\left(1-z / z_{1}\right)}, r=\left|z_{1}\right|$ and $D=\mathbf{D}\left(\rho^{-1}\left|z_{2}\right|\right)$. By the change of variable $z=z_{2} \zeta, \zeta=\xi+i \eta$, we have

$$
\begin{aligned}
H\left(\varphi_{z_{1}, z_{2}},\left|z_{1}\right|, \mathbf{D}\left(\rho^{-1}\left|z_{2}\right|\right)\right) & =\iint_{\left\{|\zeta|<\frac{1}{\rho}\right\}} \frac{\left|z_{2}\right|^{2 q-2}|\zeta|^{2 q-2}\left(1+\frac{\left|z_{2}\right||\zeta|}{\left|z_{1}\right|}\right)^{s(q-1)}}{\left|z_{2} \zeta\left(z_{2} \zeta-z_{2}\right)\left(1-\frac{z_{2} \zeta}{z_{1}}\right)\right|^{q}}\left|z_{2}\right|^{2} d \xi d \eta \\
& \leq \frac{(1+\rho)^{s(q-1)}}{(1-\rho)^{q}} \iint_{\left\{|\zeta|<\frac{1}{\rho}\right\}} \frac{|\zeta|^{q-2}}{|\zeta-1|^{q}} d \xi d \eta=: H_{1}(\rho) .
\end{aligned}
$$

The last integral converges, because its integrand has order $|\zeta|^{q-2}$ near $\zeta=0$ with $q-2>-2$ and order $|\zeta-1|^{-q}$ near $\zeta=1$ with $-q>-2$.

Similarly, setting either $z=z_{1} \zeta$ or $z=z_{2} \zeta$, we have

$$
\begin{aligned}
& H\left(\varphi_{z_{1}, z_{2}},\left|z_{1}\right|, \mathbf{D}^{*}\left(\rho\left|z_{1}\right|\right)\right) \\
& =\iint_{\{|\zeta|>\rho\}} \frac{\left|z_{1}\right|^{q}\left|z_{1}\right|^{2 q-2}|\zeta|^{2 q-2}(1+|\zeta|)^{s(q-1)}}{\left|z_{1}^{3} \zeta^{2}\left(1-\frac{z_{2}}{z_{1} \zeta}\right)(\zeta-1)\right|^{q}}\left|z_{1}\right|^{2} d \xi d \eta \\
& \leq \frac{1}{(1-\rho)^{q}} \iint_{\{|\zeta|>\rho\}} \frac{(1+|\zeta|)^{s(q-1)}}{|\zeta|^{2}|\zeta-1|^{q}} d \xi d \eta=: H_{2}(\rho), \\
& H\left(\psi_{1},\left|z_{1}\right|, A\left(\rho^{-1}\left|z_{2}\right|, \rho\left|z_{1}\right|\right)\right) \\
& =\iint_{\left\{\frac{\left|z_{2}\right|}{\rho\left|z_{1}\right|} \leq|\zeta| \leq \rho\right\}} \frac{\left|z_{1}\right|^{2 q-2}|\zeta|^{2 q-2}(1+|\zeta|)^{s(q-1)}}{\left|z_{1}^{2} \zeta\left(1-\frac{z_{2}}{z_{1} \zeta}\right)(\zeta-1)\right|^{q}}\left|z_{1}\right|^{2} d \xi d \eta \\
& \leq \frac{(1+\rho)^{s(q-1)}}{(1-\rho)^{q}} \iint_{\{|\zeta| \leq \rho\}} \frac{|\zeta|^{q-2}}{|\zeta-1|^{q}} d \xi d \eta=: H_{3}(\rho), \\
& H\left(\psi_{2},\left|z_{1}\right|, A\left(\rho^{-1}\left|z_{2}\right|, \rho\left|z_{1}\right|\right)\right) \\
& =\iint_{\left\{\frac{1}{\rho} \leq|\zeta| \leq \frac{\rho\left|z_{1}\right|}{\left|z_{2}\right|}\right\}} \frac{\left|z_{2}\right|^{3 q-2}|\zeta|^{2 q-2}\left(1+\frac{\left|z_{2}\right||\zeta|}{\left|z_{1}\right|}\right)^{s(q-1)}}{\left|z_{2}^{3} \zeta^{2}(\zeta-1)\left(1-\frac{z_{2} \zeta}{z_{1}}\right)\right|^{q}}\left|z_{2}\right|^{2} d \xi d \eta \\
& \leq \frac{(1+\rho)^{s(q-1)}}{(1-\rho)^{2 q}} \iint_{\left\{\rho^{-1} \leq|\zeta|\right\}} \frac{1}{|\zeta|^{2}|\zeta-1|^{q}} d \xi d \eta=: H_{4}(\rho) .
\end{aligned}
$$

Again the integrals $H_{j}(\rho)$ converge, for example for $j=2$, its integrand has order $|\zeta|^{s(q-1)-2-q}$ near $\zeta=\infty$ with $s(q-1)-2-q=\frac{q}{p}(s-p)-2<-2$ and order $|\zeta-1|^{-q}$ near $\zeta=1$ with $-q>-2$. The cases of $j=3,4$ are left to the reader.

Thus by (22), (23) and (24)-(27), there exists $C^{\prime}=C^{\prime}(p, s, \rho)$ such that (18) holds. For (19), replace $\mu(z)$ in the numerator by $|\mu(z)|^{2}\left|\varphi_{z_{1}, z_{2}}(z)\right| / \varphi_{z_{1}, z_{2}}(z)$ and use $|\mu(z)|^{2} \leq|\mu(z)|$ to obtain similar estimates. In fact, we can use the same constant $C^{\prime}$. Thus Lemma 10 is proved. 
Remark. If we assume (1) in Theorem 1, then it is also possible to show (12) by estimating $\iint\left(K_{f}(z)-1\right)\left|\varphi_{z_{1}, z_{2}}(z)\right| d x d y$ which is divided into several regions defined by $|z| \geq \rho^{-1}\left|z_{1}\right|,\left|z-z_{1}\right| \leq \rho\left|z_{1}\right|,\left|z-z_{2}\right| \leq \rho\left|z_{2}\right|$ and the rest, where $0<\rho<1$ will need to be chosen small according to the target $\varepsilon$.

Proof of Lemma 11. Since $K+\frac{1}{K}+2=\frac{4}{1-\|\mu\|_{\infty}^{2}}$ and $K-\frac{1}{K}=\frac{4\|\mu\|_{\infty}}{1-\|\mu\|_{\infty}^{2}}$, the integrand in $I_{p, s}(\mu ; r)$ is bounded by both

$$
\left(\frac{K+\frac{1}{K}+2}{4}\right)\left(\frac{K-\frac{1}{K}}{4}\right)^{p-2} \frac{|\mu(z)|^{2}}{1-|\mu(z)|^{2}} \frac{1}{|z|^{2}} \quad \text { and } \quad\left(\frac{K-\frac{1}{K}}{4}\right)^{p} \frac{1}{|z|^{2}\left(\frac{|z|}{r}\right)^{s}} .
$$

Integrating over $\left\{|z|<r^{\prime}\right\}$ and $\left\{|z| \geq r^{\prime}\right\}$, we immediately obtain (20). Hence $I_{p, s}(\mu ; r)$ is finite by the assumption $(2)$.

One can make the first term of the right hand side of (20) small by choosing $r^{\prime}$ small, then make the second term small by choosing $r$ even smaller. Therefore $\lim _{r \searrow 0} I_{p, s}(\mu ; r)=0$.

\section{Appendix A. Proof of Theorem 6: Grötzsch-type inequality}

We prove Theorem 6 closely following Ahlfors [A1, Chap. III.D], but improving the detail. The difference is that we do not replace $|1+\tilde{\mu}|$ by $1+|\tilde{\mu}|$ in (29) below.

Given $\left(z_{1}, z_{2}, z_{3}, z_{4}\right)$, there exist $\tau \in \mathbf{C}$ with $\operatorname{Im} \tau>0$ and a holomorphic branched double covering $p: \mathbf{E}_{\tau} \rightarrow \widehat{\mathbf{C}}$ branching over these four points, where $\mathbf{E}_{\tau}=\mathbf{C} /(\mathbf{Z}+\mathbf{Z} \tau)$. (If $z_{4}=\infty, p$ can be taken as the Weierstrass $\wp$-function.) Then $p(w)$ satisfies $p^{\prime}(w)^{2}=c \prod_{j}\left(p(w)-z_{j}\right)=\frac{c}{\varphi(p(w))}$ for some $c \in \mathbf{C} \backslash\{0\}$. There exist a counterpart $\hat{p}: \mathbf{E}_{\tau^{\prime}} \rightarrow \widehat{\mathbf{C}}$ for $\left(z_{1}^{\prime}, z_{2}^{\prime}, z_{3}^{\prime}, z_{4}^{\prime}\right)$, and a lift $\tilde{f}: \mathbf{E}_{\tau} \rightarrow \mathbf{E}_{\tau^{\prime}}$, sending generators $1, \tau$ to $1, \tau^{\prime}$ and satisfying $f \circ p=\hat{p} \circ \tilde{f}$. For $z=\varphi(w), \mu=\mu_{f}, \tilde{\mu}=\mu_{\tilde{f}}$, we have $|\varphi(z)| d x d y=|\varphi(p(w))|\left|p^{\prime}(w)\right|^{2} d u d v=|c| d u d v$ and $\tilde{\mu}(w) \frac{c}{|c|}=\mu(p(w)) \frac{\frac{p^{\prime}(w)}{p^{\prime}(w)}}{\text {. }}$ $\frac{p^{\prime}(w)^{2} \varphi(p(w))}{\left|p^{\prime}(w)^{2} \varphi(p(w))\right|}=\mu(z) \frac{\varphi(z)}{|\varphi(z)|}$. Therefore the double cover $p$ gives

$$
\bar{K}_{f}\left(z_{1}, z_{2}, z_{3}, z_{3}\right)=\sup _{\theta \in \mathbf{R}} K_{\tilde{f}, \theta}, \quad \text { where } K_{\tilde{f}, \theta}=\frac{\iint_{\mathbf{E}_{\tau}} \frac{\left|1+e^{i \theta} \tilde{\mu}(w)\right|^{2}}{1-|\tilde{\mu}(w)|^{2}} d u d v}{\operatorname{Area}\left(\mathbf{E}_{\tau}\right)},
$$

and $\operatorname{Area}\left(\mathbf{E}_{\tau}\right)=\iint_{\mathbf{E}_{\tau}} d u d v=\operatorname{Im} \tau$.

We now follow the standard Grötzsch argument: The map $\tilde{f}$ sends each horizontal curve on $\mathbf{E}_{\tau}$ to a closed curve homotopic to a horizontal curve in $\mathbf{E}_{\tau^{\prime}}$. Since $\tilde{f}$ is absolutely continuous along almost all horizontal lines, we have $\int_{0}^{1}\left|\tilde{f}_{u}(u+i v)\right| d u \geq 1$ for a.a. $v$. By integrating over $v \in[0, \operatorname{Im} \tau]$ and using $\tilde{f}_{u}=\tilde{f}_{w}+\tilde{f}_{\bar{w}}=(1+\tilde{\mu}) \tilde{f}_{w}$, we have

$$
\operatorname{Im} \tau \leq \iint_{\mathbf{E}_{\tau}}\left|(1+\tilde{\mu}) \tilde{f}_{w}\right| d u d v
$$

Cauchy-Schwarz inequality together with Jac $\tilde{f}=\left|\tilde{f}_{w}\right|^{2}-\left|\tilde{f}_{\bar{w}}\right|^{2}=\left|\tilde{f}_{w}\right|^{2}\left(1-|\tilde{\mu}|^{2}\right)$ implies

$$
\operatorname{Im} \tau^{2} \leq \iint_{\mathbf{E}_{\tau}} \operatorname{Jac} \tilde{f} d u d v \iint_{\mathbf{E}_{\tau}} \frac{|1+\tilde{\mu}|^{2}}{1-|\tilde{\mu}|^{2}} d u d v \leq \operatorname{Im} \tau^{\prime} \iint_{\mathbf{E}_{\tau}} \frac{|1+\tilde{\mu}|^{2}}{1-|\tilde{\mu}|^{2}} d u d v .
$$

Hence we have $\operatorname{Im} \tau \leq K_{\tilde{f}, 0} \operatorname{Im} \tau^{\prime}$, which means that $\tau$ is not contained in the open horodisk which is tangent to $\partial \mathbf{H}$ at $\infty$ and has distance $\log K_{\tilde{f}, 0}$ to $\tau^{\prime}$. 
If we change the generators of $\mathbf{Z}+\mathbf{Z} \tau$ from $1, \tau$ to $c \tau+d$, $a \tau+b$ with $A=$ $\left(\begin{array}{ll}a & b \\ c & d\end{array}\right) \in S L(2, \mathbf{Z})$, then we obtain an estimate on $\operatorname{Im} \frac{a \tau+b}{c \tau+d}$ and $\operatorname{Im} \frac{a \tau^{\prime}+b}{c \tau^{\prime}+d}$, and it has an effect of rotating the horizontal axis and $\tilde{\mu}$ in the integral should be replaced by $e^{i \theta} \tilde{\mu}$ for some $\theta \in \mathbf{R}$. Thus we obtain $\operatorname{Im} \frac{a \tau+b}{c \tau+d} \leq K_{\tilde{f}, \theta} \operatorname{Im} \frac{a \tau^{\prime}+b}{c \tau^{\prime}+d}$, which means $\tau$ is not in the open horodisk which is tangent to $\partial \mathbf{H}$ at $A^{-1}(\infty)=-\frac{d}{c}$ and has distance $\log K_{\tilde{f}, \theta}$ to $\tau^{\prime}$. If $\tau$ had distance greater than $\log \sup _{\theta \in \mathbf{R}} K_{\tilde{f}, \theta}$ from $\tau^{\prime}$, then $\tau$ would be in one of the horodisks as above, because $A^{-1}(\infty)(A \in S L(2, \mathbf{Z}))$ are dense on $\partial \mathbf{H}$. Hence we conclude that $d_{\mathbf{H}}\left(\tau, \tau^{\prime}\right) \leq \log \bar{K}_{f}\left(z_{1}, z_{2}, z_{3}, z_{3}\right)$. Finally the cross-ratio $\operatorname{Cr}\left(z_{1}, z_{2}, z_{3}, z_{4}\right)=\lambda(\tau)$ as a function of $\tau$ is the elliptic modular function $\lambda: \mathbf{H} \rightarrow \Omega$, which is a universal covering map. (See [A1].) Therefore $d_{\Omega}\left(\lambda(\tau), \lambda\left(\tau^{\prime}\right)\right) \leq d_{\mathbf{H}}\left(\tau, \tau^{\prime}\right)$ and Theorem 6 is proved.

\section{References}

[A1] Ahlfors, L. V.: Lectures on quasiconformal mappings. Second edition. - Univ. Lecture Ser. 38, Amer. Math. Soc., Providence, RI, 2006.

[A2] Ahlfors, L. V.: Conformal invariants: topics in geometric function theory. - McGraw-Hill, 1973.

[B1] Belinskil̆, P. P.: Behavior of a quasiconformal mapping at an isolated singular point. L'vov Gos. Univ. Uchen. Zap. Ser. Meh.-Mat. 6, 29, 1954, 58-70 (in Russian).

[B2] BeLinskIĬ, P. P.: General properties of quasiconformal mappings. - Izdat. "Nauka" Sibirsk. Otdel., Novosibirsk, 1974 (in Russian).

[BJ] Brakalova, M., and J. A. Jenkins: On the local behavior of certain homeomorphisms. Kodai Math. J. 17:2, 1994, 201-213.

[D] Drasin, D.: On the Teichmüller-Wittich-Belinskiı̌ theorem. - Results Math. 10:1-2, 1986, $54-65$.

[GL] Gardiner, F.P., and N. Lakic: Quasiconformal Teichmüller theory. - Math. Surveys Monogr. 76, Amer. Math. Soc., 2000.

[GM] GutlyanskiǏ, V., and O. Martio: Conformality of a quasiconformal mapping at a point. - J. Anal. Math. 91, 2003, 179-192.

[HSS] Hubbard, J. H., D. Schleicher, and M. Shishikura: Exponential Thurston maps and limits of quadratic differentials. - J. Amer. Math. Soc. 22:1, 2009, 77-117.

[L1] Lehto, O.: On the differentiability of quasiconformal mappings with prescribed complex dilatation. - Ann. Acad. Sci. Fenn. Ser. A I Math. 275, 1960, 1-28.

[LV] Lehto, O., and K. I. ViRTAnen: Quasiconformal mappings in the plane. 2nd Edition. Springer-Verlag, Berlin, 1973.

[McM] McMullen, C. T.: Renormalization and 3-manifolds which fiber over the circle. - Ann. of Math. Stud. 142, Princeton Univ. Press, 1996.

[RW] Reich, E., and H. R. WALCZAK: On the behavior of quasiconformal mappings at a point. - Trans. Amer. Math. Soc. 117, 1965, 338-351.

[S] Schatz, A.: On the local behavior of homeomorphic solutions of Beltrami's equations. Duke Math. J. 35, 1968, 289-306.

[T] TeichmüLleR, O.: Untersuchungen über konforme und quasikonforme Abbildung, Deutsche Math. 3, 1938, 621-678.

[W] Wiтtich, H.: Zum Beweis eines Satzes über quasikonforme Abbildungen, - Math. Z. 51, $1948,275-288$. 